\title{
MODELLING AND ANALYSIS OF MECHANICAL PULLER
}

\author{
Mr.S.Madhu, Bejgam Rithish, Guguloth Sai , Maddela Deepak \\ Department of Mechanical Engineering, Guru Nanak Institute of Technology, Hyderabad
}

ABSTRACT: In this work, a simple, inexpensive, lightweight, low energy consumption mechanical extractor was developed to operate. The puller screw shaft was first designed and followed by the nut and then its collar top. Non-conventional shapes were assumed for the collars and handles. Finite element analysis was used to evaluate the stress in the arm and collar which had complex shapes. Finite element analysis reveals that pullers are less likely to fail under design working conditions. A designed 3 arm mechanical bearing puller was fabricated and used to remove many bearings stuck in the shaft. Attempting to remove a component mounted on a shaft without using the correct type of puller can be frustrating and time consuming. A snug fit, dirt, or corrosion can make the removal process difficult. Trying puller alternatives such as cutting, blow torch, and hammer poses obvious safety risks. There is also a risk of damaging the components of the machine you are trying to repair. Mechanical pullers are a great tool for removing components from different industries. Small types of pullers are widely used by technicians such as small car stores. Larger and higher tonnage models are used in heavy industry. This project, which requires the use of a puller, generally involves the maintenance of rotating equipment. Common components to remove include bearings, gears, wheels, pins, bushings, sleeves, couplers, sprockets and pulleys.

Keywords: - Mechanical puller, collar, shaft, linkage arm, Design and analysis.

\section{INTRODUCTION}

In the routine or corrective maintenance of rotating systems, a fast, reliable and non-destructive approach need to be adopted. Use of hammer and drift pins to remove bearings, gears, pulleys and other components stuck in shafts can damage the shaft or the component. Furthermore, such method of removing stuck components can be unsafe and is a form of occupational hazard. While occupational hazards have been a concern for long, harmful habits have reportedly led to injuries and other work related health issues in the automobile industry.

A puller also called an extractor, can sufficiently be used to safely remove components stuck in a shaft [1]. Development of puller for removal of components from shaft started almost a century ago. There are two types of pullers. Hydraulic and mechanical. Hydraulic puller operates on the principle of hydraulic pressure.They are easier and quicker to use, also they are suitably used when very large extraction forces are required [1]. Ai reports on the design and manufacture of hydraulic pullers. In developing countries, obtaining hydraulic pullers using local technology is complex and expensive [9]. Mechanical pullers are simple devices because they have few components and are easy to create. Screws, bolts/heads, link arms (jaws), handles. There are two or three chins. Currently, there are various chin designs. Mechanical pullers operate on the principle of power threads. In other words, it converts angular motion into linear motion and transmits power. The operating principle and design details of the power screw are described in Ref. [2].A bellaet reported the design of an electrically controlled bearing puller. They however focused on the electrical motor. Although, much have been reported on the analysis of power screws, there is dearth of information on stress analysis of other parts of a puller. The aim of this work is to develop a mechanical puller that can easily be fabricated using available local technology and investigate the stresses acting on its parts using finite element method [3]. 


\section{LITERATURE SURVEY}

This invention relates to a mechanical puller for re moving cams, gears, bearings, and the like from shafting, as well as from gear and cam transmission assemblies [2]. In fact, the present invention is used when the removal work has to be carried out at very close proximity where it is necessary to have a solid but simple and compact tool with a very firm grip and a very high pulling force [4]. So far there have been many different types of mechanical pullers of this type in general, but it has been found that any of these prior art tools can be used with parts of no any size and shape, whether hollow or solid [5]. Moreover, none of these prior art tools have been able to achieve the full adjustability present in tools incorporating the present invention, especially while maintaining their high strength and rigidity. In addition, all previous tools combine the above qualities with the ability to provide linear pulling force instead of angled pulling, which is primarily due to inability to adapt to the specific type of work required. Noted [6]. Therefore, it is an object of the present invention to provide a mechanical extractor whose arm is generally universally adjustable.Another objective of the present invention is to provide a mechanical extractor which can be adjusted to fit parts of different shapes and sizes. Another object of the present invention is to provide a mechanical extractor with separable parts which are easily assembled and disassembled [7]. Another goal of the present invention is to provide a simple and compact mechanical extractor that contains a relatively small number of parts but is capable of exerting great resistance. A further object of the present invention is provided by an improved mechanical extractor of the type described, which is easily and economically manufactured, has a rugged structure and is very efficient in operation[8].

This invention relates to a manually operated wheel puller device for removing wheels, gears, bearings and the like from shafts, axles or other assemblies to which such parts have been tightly fitted [7]. Heretofore wheel pullers were constructed with grip ping jaws that had a tendency to spring outwardly when influenced by heavy pressure which caused damage to the part being removed, and frequently slip completely off from their engaged position, thereby causing greater damage. It is, therefore, the general object of the present invention to provide means whereby the gripping jaws of this wheel puller will operate in unison and always be in parallel position, and seat squarely against the face of the part being removed, regardless of their gripping position on parts of varying sizes [8]. Another object of the present invention is to provide a wheel extraction device provided with means for maintaining a forced screw in the center of the part to be removed.Another object of the present invention is to provide an extraction device with parallel arms and jaws with links that will equalize the pressure of the jaws with the pressure maintained on the force-applying SCCW [10]. A further object of this improved wheel puller device is that the linkage is so arranged that the greater the pressure exerted on the forcing screw the tighter the gripping jaws will grip the part being removed. In view of the forging objects of the invention and other objects that arise as the description of the invention progresses, the invention exists in detail in the combinations and arrangements of the components and structures described and claimed below. Does not deviate from the spirit of the present invention without deviating from that spirit. In the case of [9], the invention disclosed in the present specification can be carried out within the scope of claims.

\subsection{PULLER}

It was created by Yuriy Sinitsa, owner of the COLLAR company that manufactures PULLER tools, Serhiy Shkot, PULLER's dog trainer and inventor, and Varvara Petrenko, a sportswoman and dog scientist[6]. Pullers are tools used to remove parts such as bearings, pulleys and gears from the shaft. They have legs, usually two or three, that go around the back or inside the room. There is also a mandatory screw centered on the end of the shaft[3]. The screw applies force to make removal simple and with little effort from the user [6].

\section{2: CLASSIFICATION OF PULLER:}


There are many types of pullers to choose from.If you select one best suited to your application you'll get the job done safely, efficiently - and without causing damage [8]. The following are the types of pullers:

a) Two jaw puller

b) Three jaw puller

c) Hydraulic puller

d) Mechanical puller

e) Internal puller

\subsubsection{TWO JAW PULLER}

The puller which consists of 2 jaws is known as 2 jaw puller. 2-jaw pullers are popular for jobs that require removal of gears from very tight spaces. The 2- jaw puller is noted for effectively handling small gears that require immense force to remove [9].

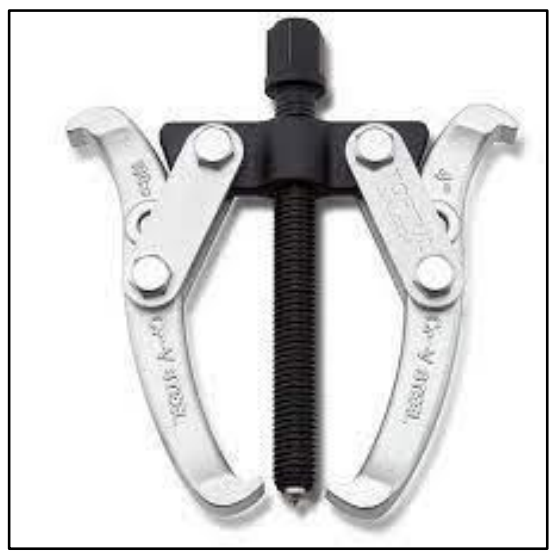

Fig 1:2 jaw puller [8]

\subsubsection{THREE JAW PULLER:}

A puller which consists of three jaws is known as 3 jaw puller. Three-jaw pullers are the most commonly used because the extra jaws help distribute the force more evenly.3-jaw pullers are commonly used for a more even pull if space permits [3].

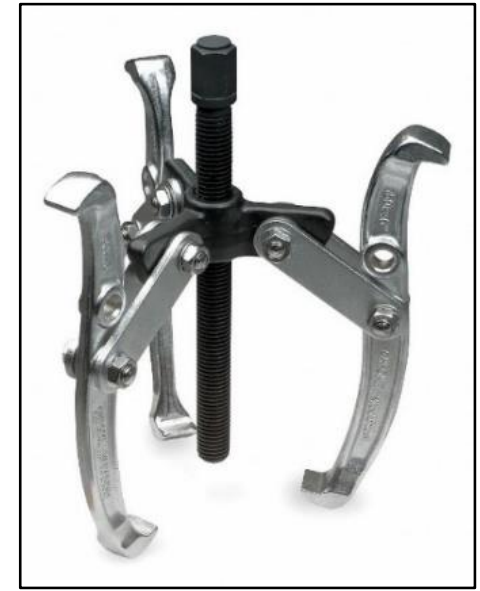

Fig 2:3 jaw puller [2]

\subsubsection{MECHANICAL PULLER:}

Mechanical puller is also called as bearing puller. Mechanical pullers work by rotating a crossbar which moves the center bolt/force screw towards the shaft [7].

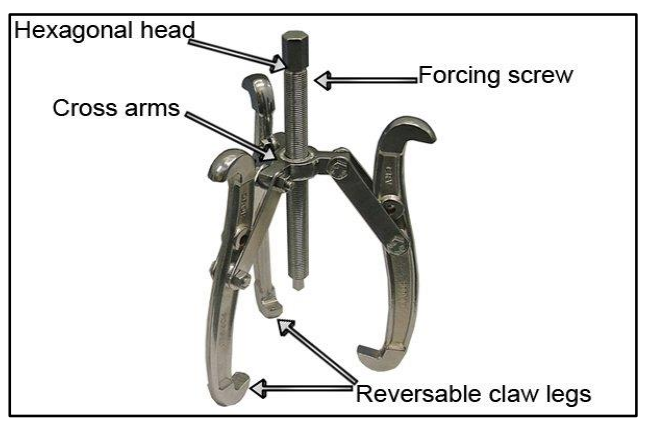

Fig 3: Mechanical puller [8]

\subsubsection{HYDRAULIC PULLER:}

Hydraulic pullers are used to remove parts (bearings or couplings) attached to the shaft. The puller uses efficiently controlled hydraulic pressure to quickly separate parts (especially compared to manual alternatives).Hydraulic pullers also do not damage the parts, which is very important when performing maintenance activities [8]. 


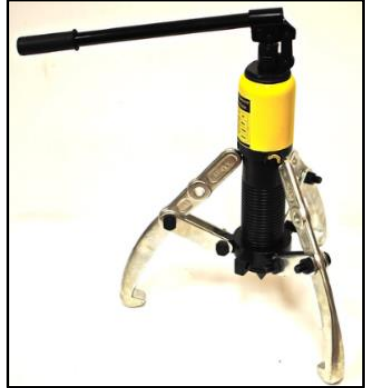

Fig 4: Hydraulic puller [10]

\subsection{5: INTERNAL PULLER}

These are used to remove parts attached to shafts when spindle support is not available. The internal puller is used with an attached sliding hammer that can apply great impact force[9].

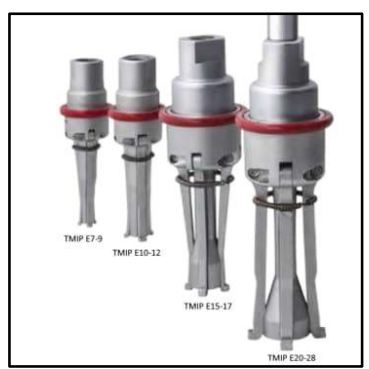

Fig 5: Internal bearing puller [9]

\subsubsection{PNEUMATIC PULLER:}

A "pneumatic bearing puller" is a device that can be operated independently and the bearing can be removed from the puller without installing the puller from the support device [5]. Therefore, special skills are required to operate or withdraw. Can be widely used. Easy to install, remove and reassemble on bearings. No workspace is required to install the puller. Can be used effectively for drawers. Here we change the bending and sheet metal removal tools for bending and sheet metal removal operations on the same machine [2].

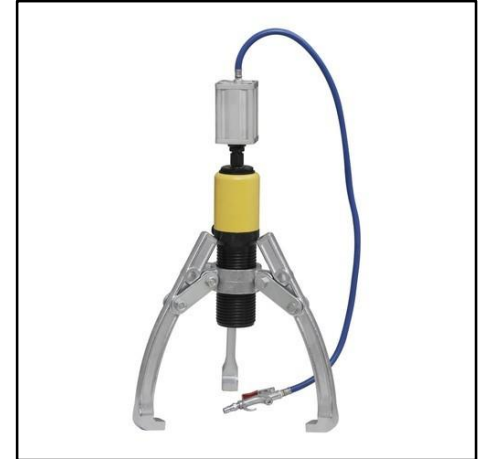

Fig 6: Pneumatic puller [10]

\subsection{WORKING OF MECHANICAL PULLER}

Mechanical pullers operate on the principle of power threads. In other words, it converts angular motion into linear motion and transmits power[12]. Steps involved in mechanical puller are

Step 1: Puller Placement

Place the puller either remotely or inside around the part (contingent upon the part being eliminated).

Step 2: Leg Adjustment

Adjust the puller legs with the goal that they fit firmly around the part.

Step 3: Position the Screw

Turn the screw clockwise until it is contacting the shaft. Change the legs again so the focal point of the screw is in the focal point of the shaft.

\section{Step 4: Turn the Screw}

Turn the screw cautiously and gradually to apply power onto the shaft.

\section{Step 5: Remove the Part}

Continue turning the screw until it pulls the piece of the shaft.

\section{Step 6: Loosen the Screw}

Loosen the screw by turning it the other way and eliminate the part from the legs of the puller

\section{Methodology}


Problem Statement

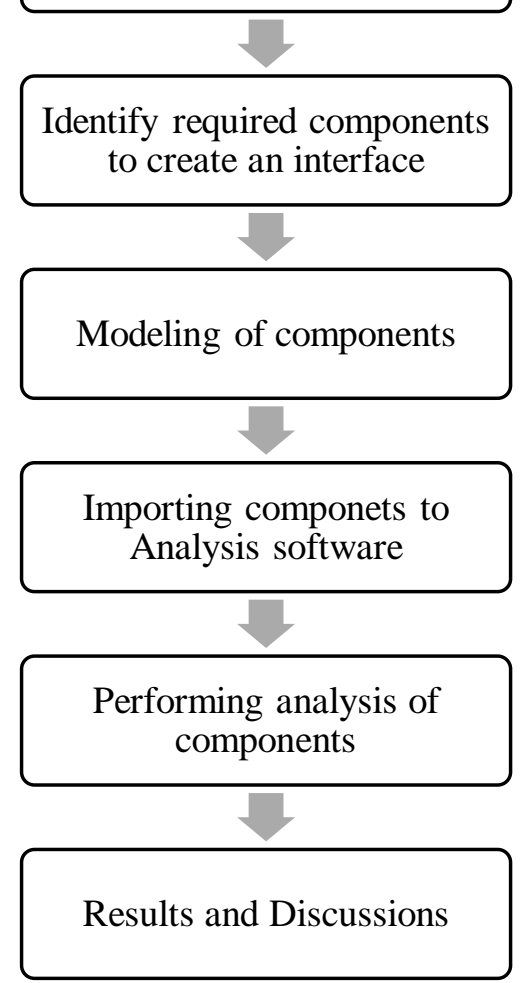

\section{PROBLEM STATEMENT}

Attempting to eliminate shaft-mounted parts without utilizing the right sorts of puller can be disappointing and tedious. Obstruction fits, soil, and erosion can make the evacuation interaction troublesome. Attempting options in contrast to pullers, like cutting, burning, or pounding presents clear of making harm the machine parts you are attempting to keep up with. Mechanical puller is incredible apparatus for eliminating parts across a wide range of modern areas. Parts to eliminate incorporate heading, gears, wheels, pins, bushings, sleeves, couplers, sprockets, and pulleys

\section{OBJECTIVE}

The various objectives of the project are listed below:

- To design the components of mechanical puller

- To perform analysis on the mechanical puller and compare the evaluated results

\subsection{Design of Mechanical Puller}

\subsubsection{DESING PROCEDURE}

Step 1: Double Click on CATIA V5 Software to Open it.

Step 2: click on start -mechanical design-part Design -to model required part

Step 3: Select required plane and create $2 \mathrm{~d}$ view of the part

Step 4: Exit the workbench in order to enter $3 d$ view and apply pad to sketch to produce $3 \mathrm{~d}$ part

Step 5: Perform the operations as required for the part

Step 6: Final 3d model is created

\subsubsection{COMPONENTS OF MECHANICAL PULLER}

- Linkage arm

- Collar

- Screw shaft

- Bolt

- Nut

- Washer

\subsubsection{Linkage arm}

The linkages work together to ensure that the rotational motion of the screw is changed to linear motion to pull the bearing out from the shaft [9]. The purpose of the arms is to support the puller and enable it to withstand compressive load exerted on it. The link is a bit complex and thus requires casting as a manufacturing process. For this reason, gray cast iron as a material is selected for the frame. Additionally, cast iron is cheap, has high compressive strength and can be used to manufacture any complex shape without involving costly machining operations [10].

\section{DESIGN AND ANALYSIS}




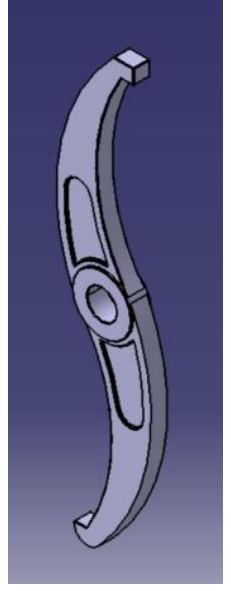

Fig 7: Linkage arm

\subsubsection{Collar}

The collar is the main component of the mechanical puller. The collar houses all the components of the mechanical puller. [11]

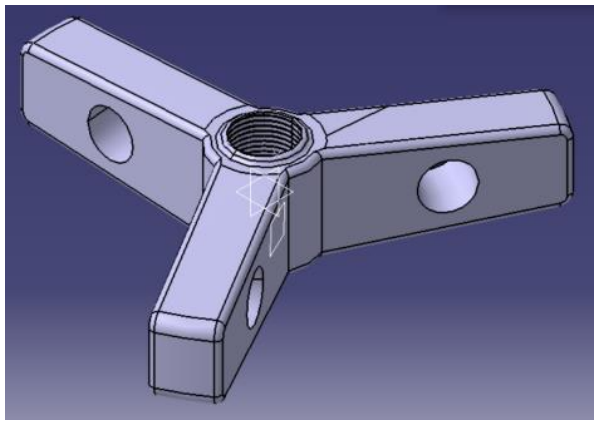

Fig 8: Collar

\subsubsection{SCREW SHAFT}

The screw is to transmit power while converting rotational motion into translational. It is to withstand torsional second, compressive power and twisting moment [5]. The square type screw profile was selected for this work due to its higher efficiency and self-locking and ease of fabrication required. The material selected is plain carbon steel because of its excellent workability, wide range of attainable combinations of strength properties and its availability [6].

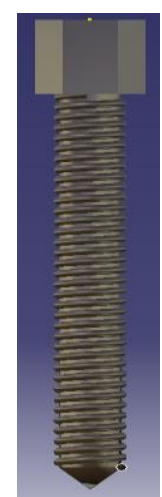

Fig 9: Screw shaft

\subsubsection{Bolt}

A bolt is a type of fastener, usually made from metal, that commonly comprises a head at one end, a chamfer at the other, and a shaft characterized by an external helical ridge Kona h rd. [11]. Bolts are normally used to hold materials or items together, or to situate objects.

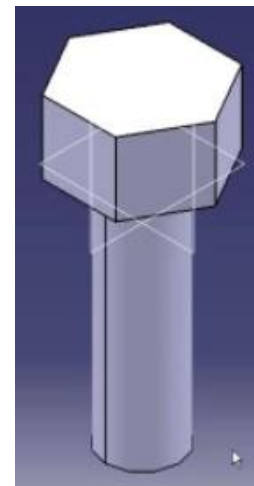

Fig 10: Bolt

\subsubsection{Nut}

There exists a general movement between the screw and the nut which causes erosion, grating thus causes wear of the material utilized for screw and nut. It was therefore necessary to select a softer material for the nut. As a result, phosphor bronze which is a copper compound with little level of lead was selected for the nut. Its advantages are: good corrosion resistance, low coefficient of friction, high tensile strength. 


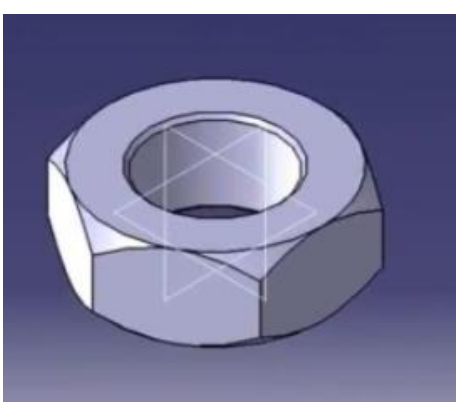

Fig 11: Nut

\subsubsection{Washer}

The washer is a mechanical machine part helpful in mix with a screw clasp. All the more explicitly, the item functions admirably alongside a screw and nut. Washers are commonly comprise of a leveled, circular, or disc shaped piece of metal. The segment has an opening in the center. Prior to appending string latches into a surface, it is fundamental for place a washer through the end. These items typically hold the screw back from relaxing or disseminating the heap from the other components such as nut or bolt over the surface area.

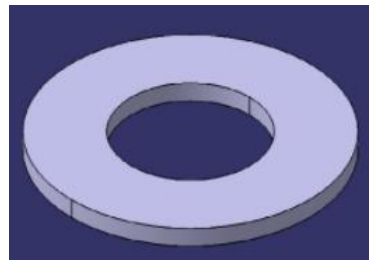

Fig 12: Washer

\subsection{ASSEMBLY OF MECHANICAL PULLER}

In order to assemble the components of mechanical puller in CATIAV5 R20. First we need to open the software by double clicking on the icon. When the software opens, on the user interface at the top left corner click on the start tab, then some list appears on the screen, click on mechanical design and then again another list of features appears select the assembly as here we are assembling the components of mechanical puller. After clicking on the assembly a product name appears on the list of trees change the name by right click and then select properties or by pressing Alt plus Enter on the keyboard. In order to import the components of puller click on product and then click on existing component in components tab and then select the component you want to import, for assembly of mechanical puller first we need to import the collar part as it houses all the components. After importing apply fix constraint to it by clicking on fix command in the connections tab to prevent the component from moving in any direction (i.e. making all the degrees of freedom as zero). Then import the screw shaft and give coincidence connection to the shaft axis with hole axis on the collar. After that import the puller connector and give coincidence connection with hole on collar. After that import linkage arm and move the component from the center by clicking on move in move tab and translate or rotate the component as per the requirement and give coincidence connection to collar hole axis with hole axis of linkage arm and also with hole on the puller connector. Similarly assemble all the components. The final assembly of mechanical puller obtained as shown.

\section{Assembly Requirements}

\begin{tabular}{|l|l|}
\hline Component & Quantity \\
\hline Linkage Arm & 3 \\
\hline Collar & 1 \\
\hline Screw Shaft & 1 \\
\hline Bolt & 6 \\
\hline Nut & 6 \\
\hline Washer & 6 \\
\hline
\end{tabular}




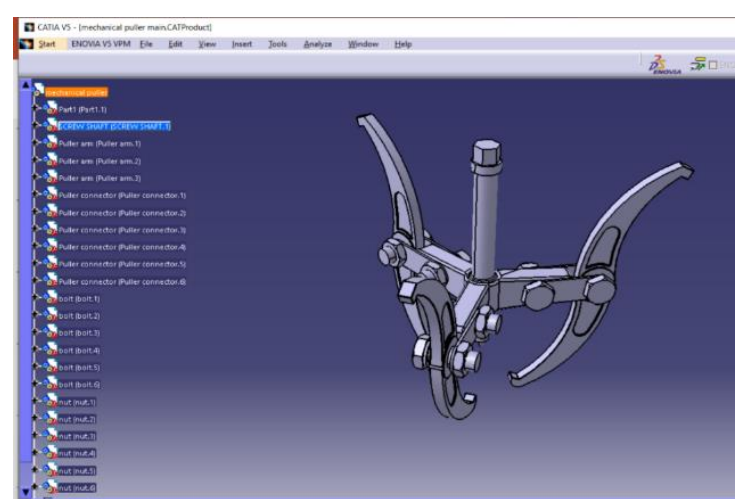

Fig 13: Assembly of Mechanical Puller

\subsection{ANALYSIS OF MECHANICAL PULLER}

The analysis is conducted on the mechanical puller assembly considering the deformations and stresses produced while removing the shaft mounted components like bearings, pulleys and gears. Here we have performed transient structural analysis on the mechanical puller as we need to apply load on the linkage arm tips while the movement of collar takes place. There are various analysis software available, here we have used ANSYS software with WORKBENCH 16.0 version to perform analysis. The part and assembly created in CATIAV5 is not supported to import into ANSYS software. It supports limited file formats like IGES, STP etc. here we have converted the assembled product into STP file format and performed analysis on it.

\section{ANALYSIS PROCEDURE}

- Step 1: Double click on WORKBENCH 16.0 to open ANSYS software. Click on import and select the part to open it.

- $\quad$ Step 2: Click on transient structural drag and place it on the Geometry.

- $\quad$ Step 3: Double click on model and then a preview of part appears on the screen

- Step 4: Now click on geometry on mechanical window to check whether required parts are imported
- Step 5: click on connections -Right click -click on joints. Now give required connections to perform analysis

- Step 6: Click on mesh, in default it shows mesh increase or decrease elements size as required. On Right side it shows number of nodes and elements created

- Step 7: Now right click on transient and give required movement for parts and click on generate solution. Finally, results are obtained by performing analysis

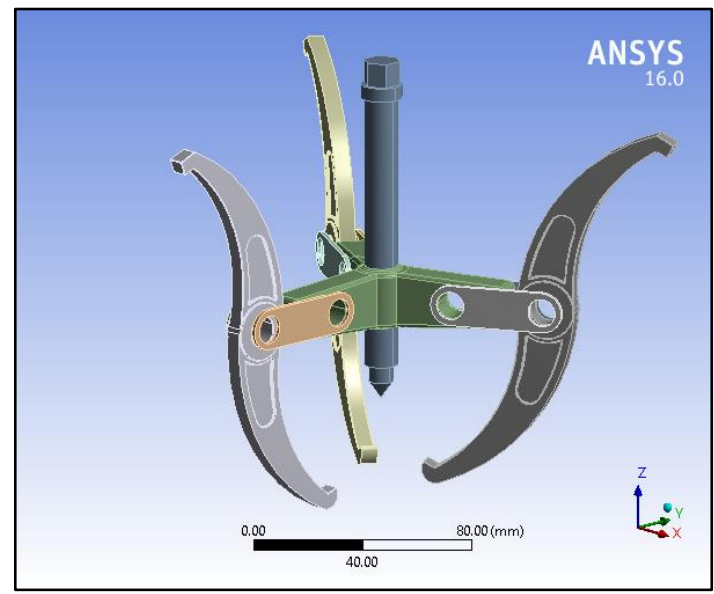

Fig 14: Imported Model of Mechanical Puller

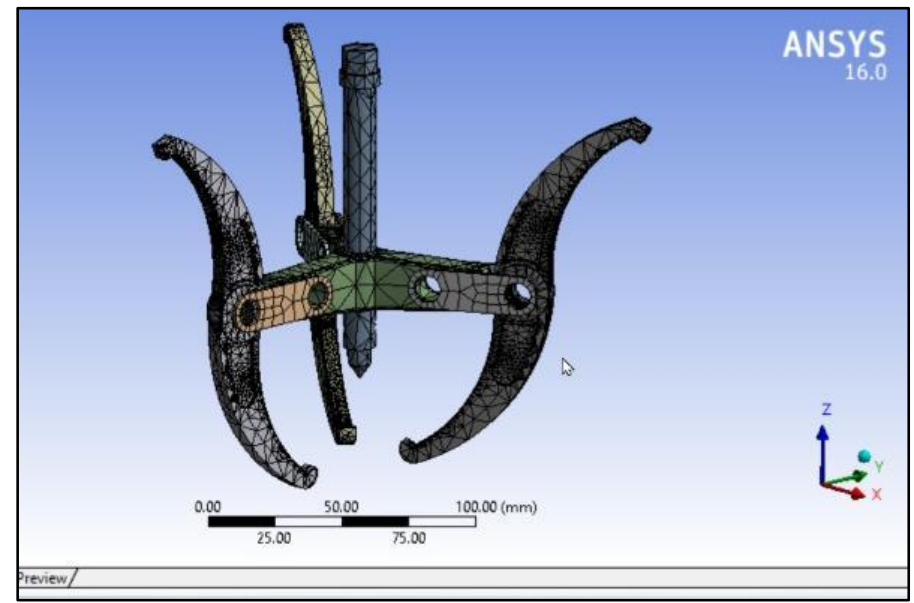

Fig 15: Mesh model of mechanical puller 


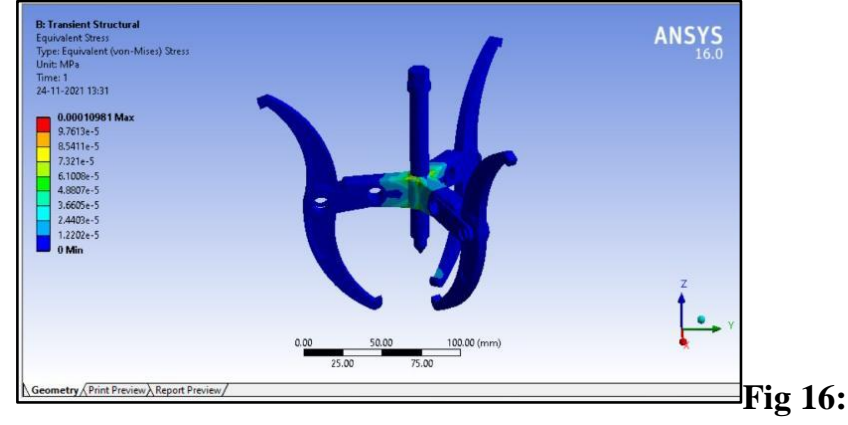

Equivalent stress of Mechanical puller

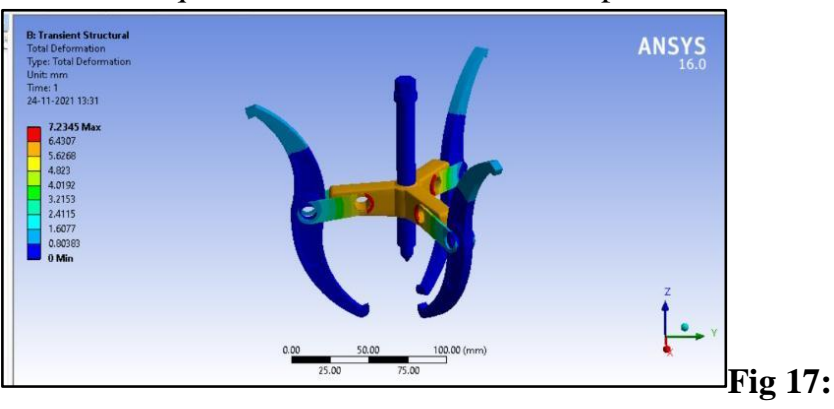

Total Deformation of mechanical puller.

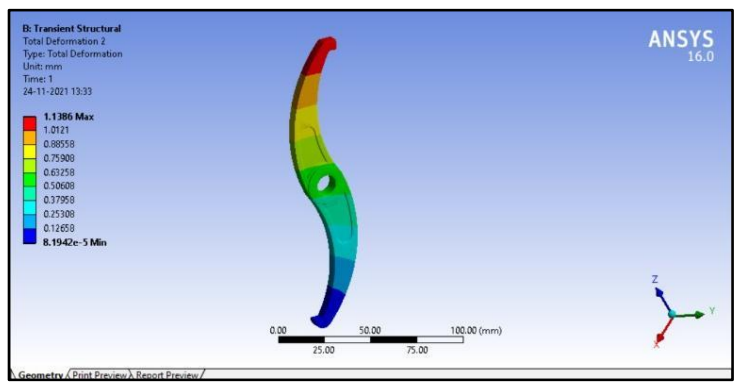

Fig 18:Total deformation of linkage arm

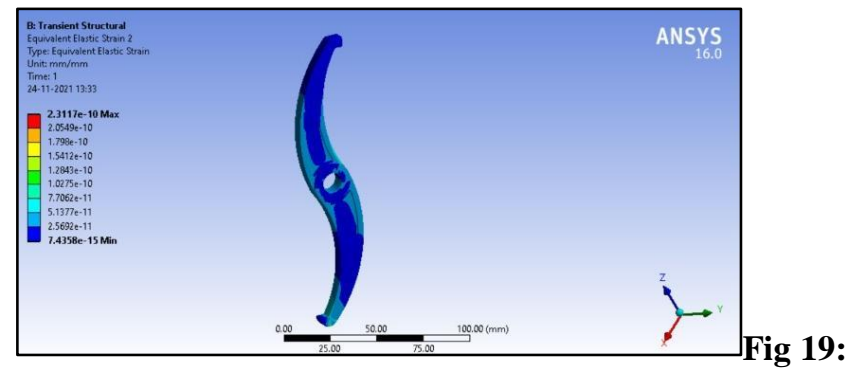

Equivalent Elastic strain of linkage arm

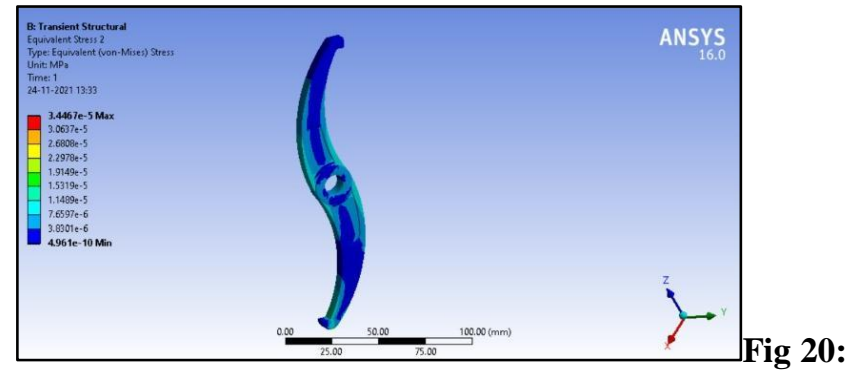

Equivalent stress of linkage arm

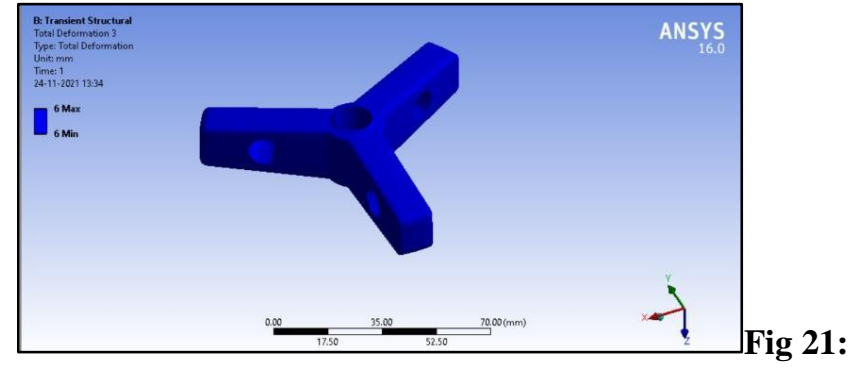

Total deformation of collar

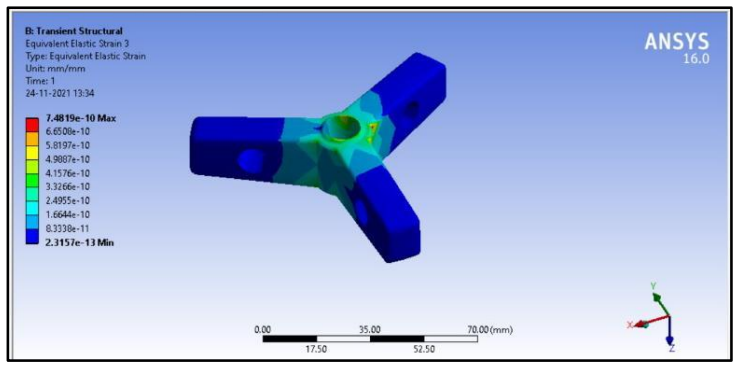

Fig 22: Equivalent elastic strain of collar

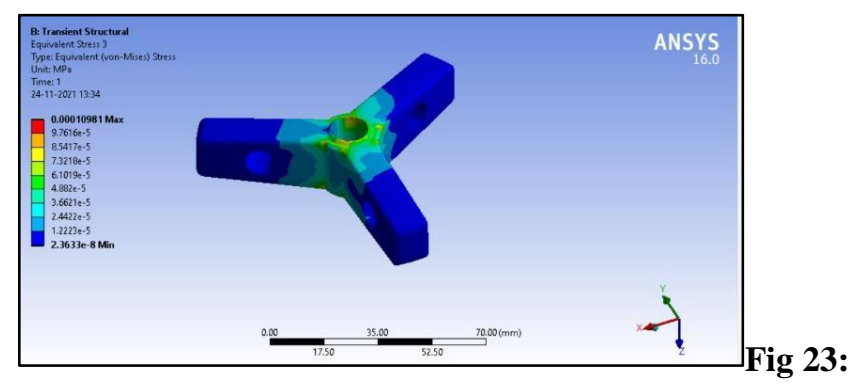

Equivalent Stress of collar

\section{Analysis Results of Mechanical Puller}

\section{Evaluated Results by Applying Magnitude}

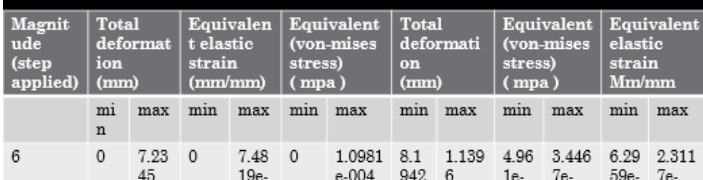

$\begin{array}{lllllllll}7.48 & 0 & 1.0981 & 8.1 & 1.139 & 4.96 & 3.446 & 6.29 & 2.311 \\ 19 \mathrm{e}- & & \mathrm{e}-004 & 942 & 6 & 1 \mathrm{e}- & 7 \mathrm{e}- & 59 \mathrm{e}- & 7 \mathrm{e}-\end{array}$ 005

$\begin{array}{lllllllllllllll}5 & 0 & 6.03 & 0 & 6.18 & 0 & 9.0396 & 5.7 & 0.801 & 5.58 & 2.608 & 6.29 & 1.751\end{array}$

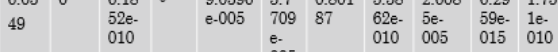

$\begin{array}{llllllllllllll}4 & 0 & 4.83 & 0 & 4.91 & 0 & 7.1497 & 3.7 & 0.520 & 3.39 & 1.840 & 3.38 & 1.235 \mathrm{e}\end{array}$

$\begin{array}{llllllll}4.91 & 7.1497 & 3.7 & 0.520 & 3.39 & 1.840 & 3.38 & 1.235 \\ 47 \mathrm{e}- & \mathrm{e}-005 & 479 & 78 & 95 \mathrm{e}- & 2 \mathrm{e}- & 32 \mathrm{e} & -010 \\ 010 & & & \mathrm{e}- & & 010 & 005 & 015\end{array}$

\begin{tabular}{l|l|l|l|l|l|l|l|l}
\hline .66 & 0 & $5.307 e$ & 2.1 & 0.297 & 2.59 & 1.162 & 2.62 & 7.797
\end{tabular}

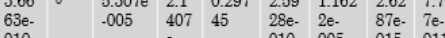
$010 \quad 005$

$\begin{array}{lllllllll}2.43 & 0 & 3.5057 & 9.6 & 0.134 & 1.04 & 6.110 & 1.09 & 4.090\end{array}$

$\begin{array}{llllllll}51 \mathrm{e}- & \mathrm{e}-005 & 667 & 32 & 38 \mathrm{e}- & 6 \mathrm{e}- & 28 \mathrm{e}- & 5 \mathrm{e} . \\ 010 & & \mathrm{e}-\mathrm{e} & & 010 & 006 & 015 & 011\end{array}$ 


\section{Conclusion}

We designed a mechanical 3-arm puller and analyzed the stress on the puller and its irregularly shaped parts using the finite element method. The designed prototype model was used and tested to remove bearings and pulleys attached to the shaft, resulting in satisfactory performance. Finite element analysis of mechanical pullers, puller arms, and collars showed that pullers are less prone to failure under expected working conditions.

\section{Future scope}

The Mechanical Pullers market report contains a comprehensive study of crucial aspects such as key trends and opportunities in this industry vertical that can promise substantial returns to stakeholders in the forthcoming years. It also outlines the strategies that can be adopted by businesses to address the current and upcoming challenges.

\section{REFERENCES}

[1]. International Journal of Innovative Science and Research Technology ISSN No: -2456-2165.

[2]. KUKKO-Werkzeugfabrik Kleinbongartz \& Kaiser oHG. General Catalouge. KUKKO. [Online] 2016. [Cited: May 20, 2018.] www.KUKKO.com.

[3]. Budynas-Nisbett.Shirley's Mechanical Engineering Design. 8th. United States: The McGraw-Hill Companies, 2008. ISBN: 0-390-76487-6.
[4]. Schaeffler Technologies AG \& Co. Kg. Mounting and Maintenance of Rolling bearing. Schaeffler. [Online] April 2018. [Cited: May 20, 2018.] www.schaeffler.com/services.

[5]. Work Habits and Health Problems of Automobile Technicians at Mechanic Village, Uyo,

Nigeria. Johnson, Ofonime Effiong and Bassey, Emem Anietie. 5, s.l.: Global Advanced Research Journal of Medicine and Medical Sciences, 2016, Global Advanced Research Journal of Medicine and Medical Sciences, Vol. 5, pp. 136-142. ISSN: 2315-5159

[6]. "DOG PULLER Championship History, 2018

Highlights and 2019 Details. - Free Online

Library" www.thefreelibrary.com. Retrieved 2020-02-07.

[7]. Vahid-Araghi, O and Golnaeaghi, F.Friction Induced Vibration in Lead Screw drive. s.l.: Springer, 2011. ISBN: 978-1-4419-1751-5.

[8] United States Patent Office 3,063,140 Patented Nov. 13, 1962

[9] United States Patent Office Patented Feb. 4, 1958

[10]. Visit Enerpac.com

[11]. tengtoolsusa.com

[12]. Indiamart.com 